\title{
Successful reversal of isolated delayed spinal cord ischemia following endovascular abdominal aneurysm repair
}

This article was published in the following Dove Press journal:

Vascular Health and Risk Management

\author{
Qusai Aljarrah' \\ Mamoon H Al-Omari ${ }^{2}$ \\ Mwaffaq Elheis ${ }^{2}$ \\ Mooath Al-Jarrah ${ }^{3}$ \\ Abdelwahab Jamal ${ }^{3}$ \\ Abdullah Alzoubi ${ }^{4}$ \\ 'Department of Surgery, Faculty of \\ Medicine, Jordan University of Science \\ and Technology, Irbid 22II0, Jordan; \\ ${ }^{2}$ Department of Diagnostic and \\ Interventional Radiology, Faculty of \\ Medicine, Jordan University of Science \\ and Technology, Irbid 22II0, Jordan; \\ ${ }^{3}$ School of Medicine, Faculty of Medicine, \\ Jordan University of Science and \\ Technology, Irbid 22 I I0, Jordan; \\ ${ }^{4}$ Department of Pharmacology, Faculty of \\ Medicine, Jordan University of Science \\ and Technology, Irbid 22I10, Jordon
}

\begin{abstract}
A 74-year-old patient presented with isolated fecal incontinence 6 weeks following endovascular aneurysm repair. The delayed presentation of spinal cord ischemia was precipitated by commencement of alpha-blockers for benign prostatic hyperplasia. This case stresses that vulnerability to spinal cord perfusion is not limited to the perioperative period. In addition, systemic arterial pressure should be closely monitored in cases of marginal vascular insufficiency of the spinal cord.
\end{abstract}

Keywords: abdominal aortic aneurysm, endovascular aneurysm repair, spinal cord ischemia, fecal incontinence

\section{Introduction}

Spinal cord ischemia (SCI) remains a rare, but devastating complication following abdominal aortic aneurysm (AAA) repair. Understanding of the complex pathophysiology of SCI is evolving, but the inciting factors behind many cases remain unidentified. We herein report a case of delayed fecal incontinence due to SCI following EVAR, successfully treated with complete resolution of the patient's symptoms. Our case suggests that vulnerability to spinal cord perfusion is not limited to the perioperative period, but persists following patient discharge, and that vigilant attention to systemic arterial pressure is crucial in cases of marginal vascular insufficiency of the spinal cord.

\section{Case report}

A 74-year-old male patient presented to our hospital with isolated fecal incontinence and loose stool 6 weeks following EVAR. The patient had a notable history of coronary heart disease, previous coronary artery bypass surgery, dyslipidemia, and a history of ulcerative colitis. He was initially referred to our vascular service by the neurosurgery team after a lumbar magnetic resonance imaging (MRI), performed to investigate lower back pain, revealed a large AAA. The MRI also revealed multiple degenerative intervertebral disc bulges in the L2-S1 segments, with significant secondary spinal canal stenosis (Figure 1).

Initial vascular assessment revealed an expansile pulsation in the epigastric region associated with mild tenderness and a prominent pulsation in the right iliac fossa. Peripheral vascular examination was otherwise unremarkable.
Correspondence: Qusai Aljarrah

Vascular Surgery, Department of Surgery, Faculty of Medicine, Jordan University of Science and Technology, Irbid 22110, Jordan

Tel +96277559 3131

Fax +9 6227201064

Email qmaljarrah@just.edu.jo 


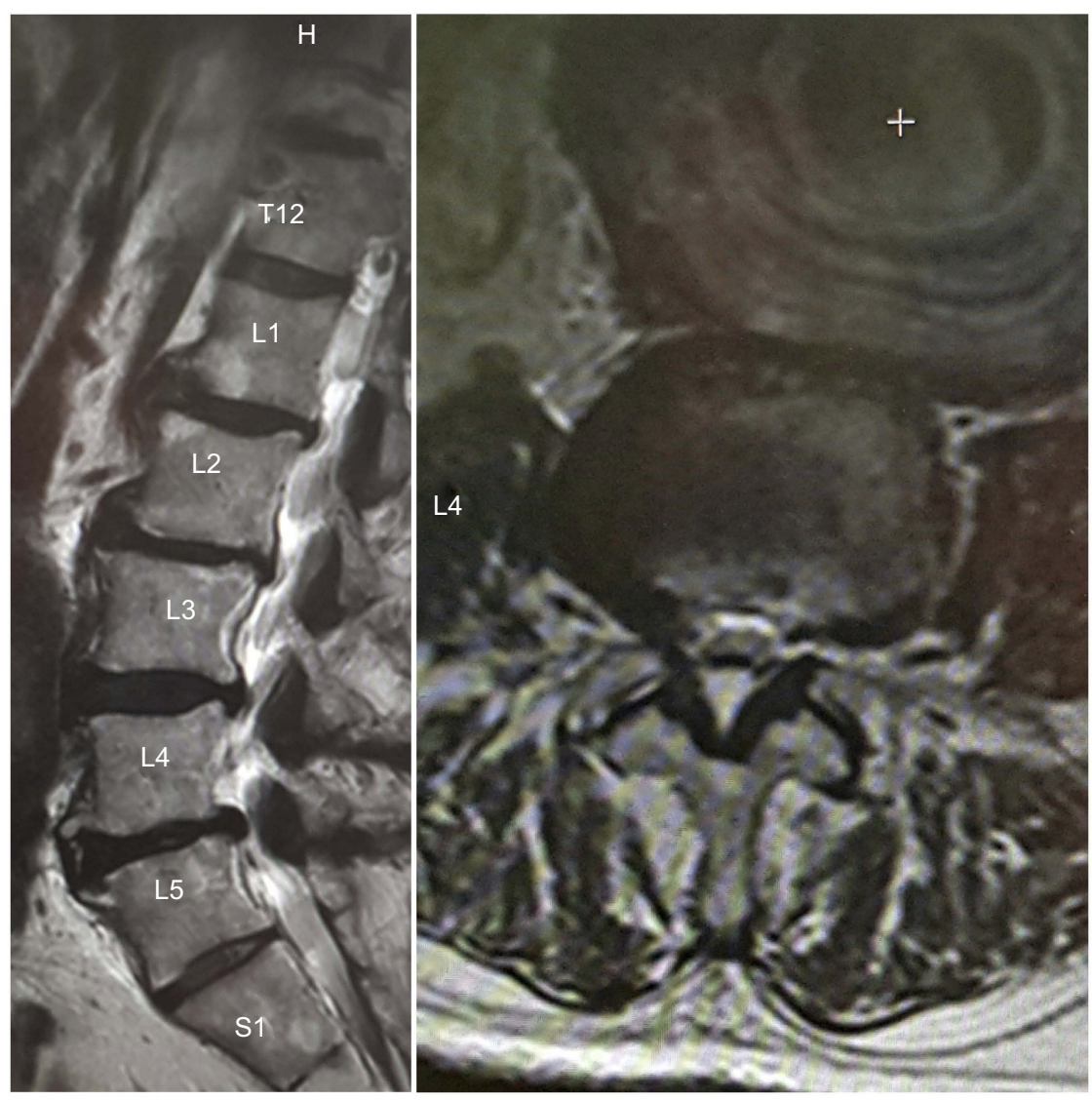

Figure I Lumbar Spine MRI: (A) sagittal T2WI showing degenerative disc changes with significant thecal sac compression at levels L5-SI, Magnification XI (B) axial T2W at levels L4-L5 showing a massive AAA, Magnification X2.

Abbreviations: AAA, Abdominal Aortic Aneurysm; MRI, Magantic Resonance Imaging.

A computed tomography angiogram (CTA) revealed a $10 \mathrm{~cm}$ infrarenal inflammatory AAA, a $3 \mathrm{~cm}$ aneurysmal right common iliac artery, and an ectatic $2 \mathrm{~cm}$ left common iliac artery. He was found to have a favorable aortic neck anatomy, and a sufficient landing zone (Figure 2). The patient underwent urgent EVAR with a Medtronic Endurant device (Medtronic Vascular, Santa Rosa, CA, USA). We selectively embolized the right internal iliac artery, and intentionally covered both internal iliac arteries due to the features seen on the CTA (Figure 3).

The patient's postoperative course was uneventful, leading to his discharge on postoperative day three. Two weeks later, he was seen in the outpatient clinic and was found to be recovering adequately with sufficiently healing wounds. Furthermore, a CTA 4-week postoperation showed complete exclusion of the aneurysm without endoleak, as well as maintenance of pelvic perfusion via retrograde flow from the left internal iliac artery (Figure 4).

Six weeks post-EVAR, the patient experienced an episode of urinary retention due to obstructive uropathy (secondary to benign prostatic hyperplasia), for which he was prescribed Alfuzosin. Following initiation of Alfuzosin, the patient began having dizzy spells and postural hypotension. Two weeks later, he began complaining of loose stool and fecal incontinence, at which time he presented to the vascular clinic.

On clinical evaluation, the patient was hypotensive, with a blood pressure (BP) of 77/44 mmHg. Digital rectal exam (DRE) revealed laxity of the anal sphincter and soft stool, without blood or mucus. A neurological exam indicated 5/5 power in both lower extremities, intact deep tendon reflex, and unremarkable sensory examination. An urgent colonoscopy revealed mild inflammation in the rectum, with a biopsy showing features of ischemic colitis (Figure 5). We consulted the neuromedicine team, who suggested the possibility of isolated SCI, and advised a new lumbosacral spine MRI. This MRI showed no evidence of acute ischemia, and no changes when compared to his initial scan.

The patient was admitted for 5 days of intensive therapy to improve his BP. He was started on IV fluids, and Alfuzosin was ceased. A daily $16 \mathrm{mg}$ dose of IV dexamethasone was initiated for suspected SCI, as well as loperamide to thicken 

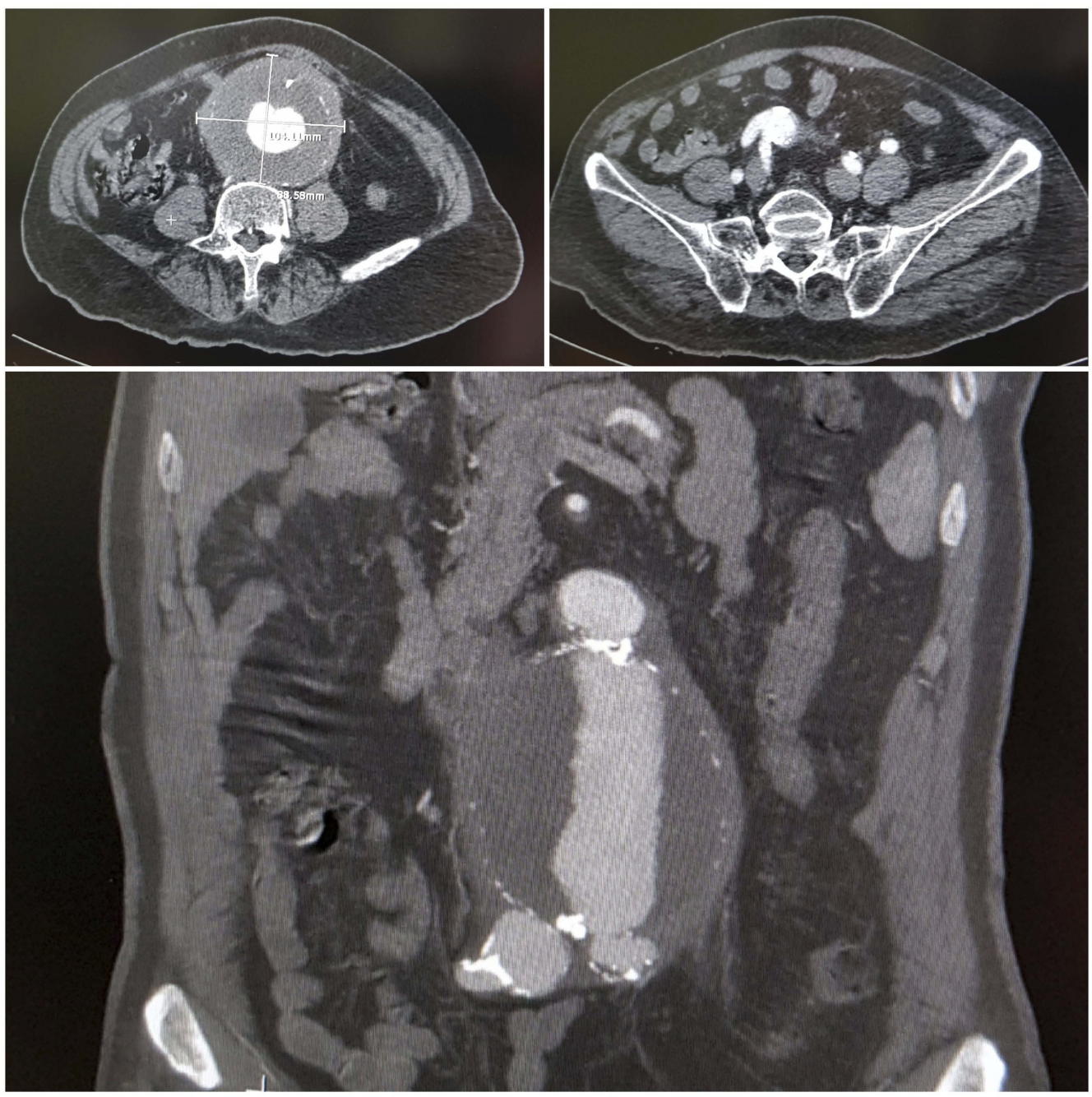

Figure 2 Preoperative CTA: (A) axial view showing a thick-walled $10 \mathrm{~cm}$ infrarenal AAA, Magnification $\times$ I (B and C) Axial (Magnification XI) and coronal (Magnification $\mathrm{X}$ ) views showing an aneurysmal extension into to right IIA.

Abbreviations: AAA, Abdominal Aortic Aneurysm; CTA, Computed Tomography Angiography.

his stool. After treatment, his BP rose to an average of $110 / 60$ $\mathrm{mmHg}$, and the patient reported significant improvement in sphincter control. Further DREs revealed greatly improved anal tone. Based on these findings, the patient was discharged and prescribed fludrocortisone $(0.1 \mathrm{mg} /$ day $)$, clopidogrel (75 mg), aspirin (100 mg), enoxaparin (40 mg), vitamin E, and loperamide ( $2 \mathrm{mg})$.

Two weeks following discharge, the patient was seen in the outpatient clinic, where examination showed a normal BP of $115 / 60 \mathrm{mmHg}$, complete recovery of sphincter control, and resolution of diarrheal symptoms.

\section{Discussion}

$\mathrm{SCI}$ is a potentially disastrous complication following EVAR. SCI can either present immediately or in a delayed fashion. ${ }^{1}$ Neurological deficits can be transient or permanent. ${ }^{2}$ The reported incidence of SCI following EVAR is marginally less than that following open repair. ${ }^{3}$ Luckily, the incidence of SCI following aortic pathologies has recently declined, presumably due to the development of advanced endovascular techniques and improved understanding of variables affecting spinal cord circulation. ${ }^{4}$ In a recent systematic review, Moulakais et al identified 25 cases of SCI following elective EVAR and found that only $15 \%$ of the reported cases presented with delayed SCI. ${ }^{1}$ Additionally, recovery rates remained low, with only $25 \%$ of the cohort experiencing complete recovery of neurological deficits. ${ }^{1}$

The mechanism of SCI appears to be a consequence of multiple complex interactions, and our current understanding is derived from thoracic and thoracoabdominal aortic surgeries. EVAR and open repair result in different mechanisms of SCI, including endovascular exclusion of 


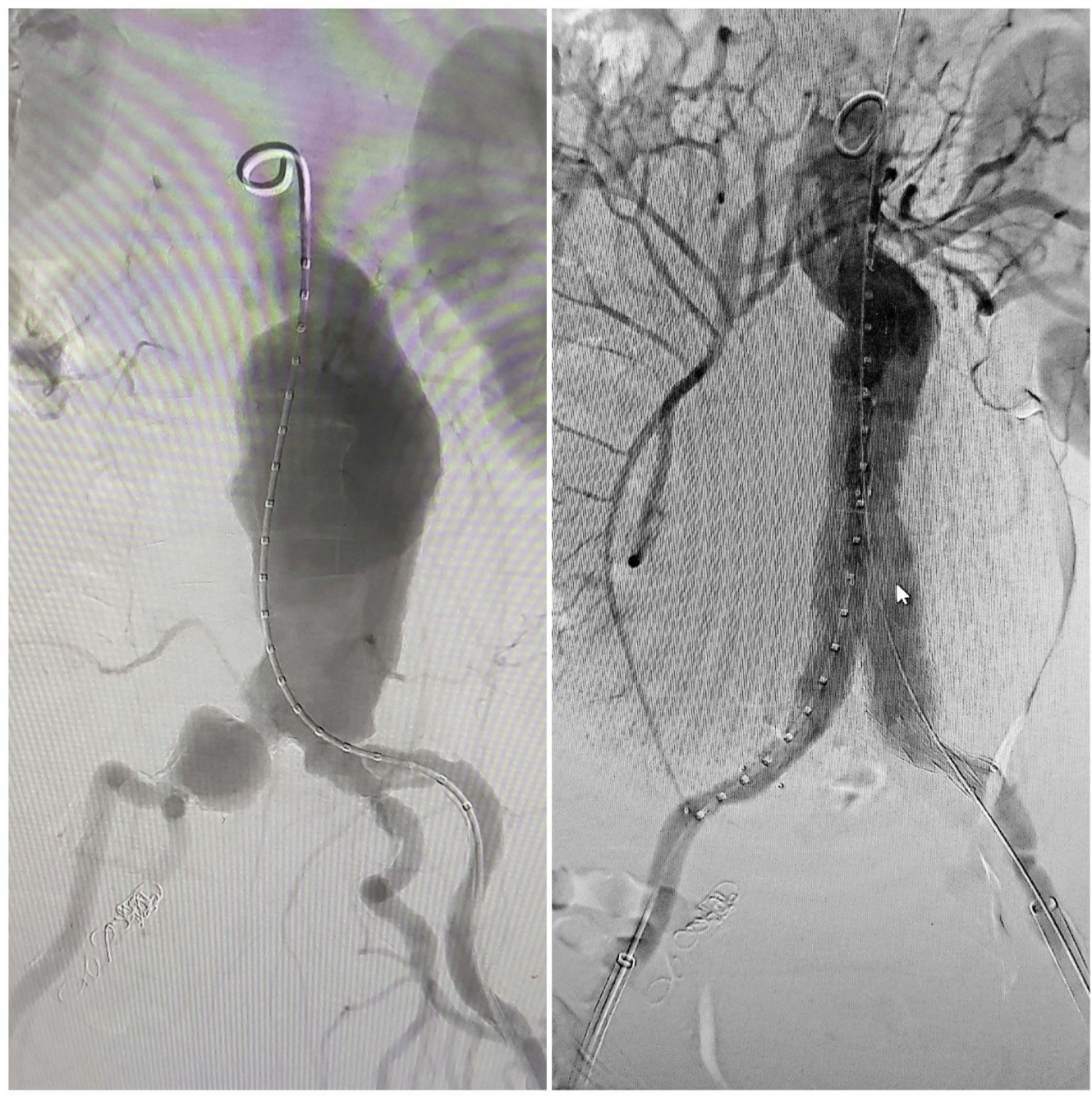

Figure 3 Intraoperative digital subtraction angiogram: (A) large infrarenal AAA with aneurysmal dilatation of right common iliac artery and short ectatic left common iliac artery, Magnification XI(B) successful deployment of the bifurcated graft and embolization of RIIA, Magnification XI.

Abbreviations: AAA, Abdominal Aortic Aneurysm; RIIA, Right Internal lliac Artery.

vital arterial supply to the spinal cord, perioperative hypotensive episodes, and atheroembolization of debris into pelvic circulation following endovascular instrumentation. ${ }^{5,6,7}$ Three important mechanisms contribute to delayed SCI; reperfusion injury with the flux of inflammatory and neurochemical mediators, spinal cord edema, and lastly arterial hypotension., ${ }^{7,8}$ Recently, Awad et al unveiled the importance of spinal cord edema in the development of SCI, suggesting a multifactorial process of development. ${ }^{10}$ Our understanding of SCI is continuously evolving and most likely multifactorial.

Understanding the anatomical blood supply to the spinal cord is fundamental for identifying the etiology of SCI. The spinal cord is not only reliant on a key feeding artery, but also on a rich network of collaterals from branches of the subclavian and vertebral arteries proximally, branches of the intercostal and lumbar arteries centrally, and branches of the inferior mesenteric artery, internal iliac artery, and sacral arteries distally. ${ }^{2,11}$ These important collateral networks can be interrupted or sacrificed by aortic pathology, or the treatment modality used to correct it. Furthermore, spinal cord autoregulation is disrupted when aortic treatment is performed. ${ }^{4}$ Preoperative CT and MRI scans must be carefully reviewed to identify potential risks of spinal cord arterial compromise, to identify vulnerable patients, and guide the course of treatment. ${ }^{4,11}$

SCI has an unpredictable clinical presentation with some patients presenting with varying degrees of lower limb paresis or paralysis, accompanied in some cases with fecal and/ or urinary incontinence. Nishioka et al reported a case of isolated bladder and rectal incontinence following EVAR, with complete recovery after hyperbaric oxygen therapy. ${ }^{12}$ 


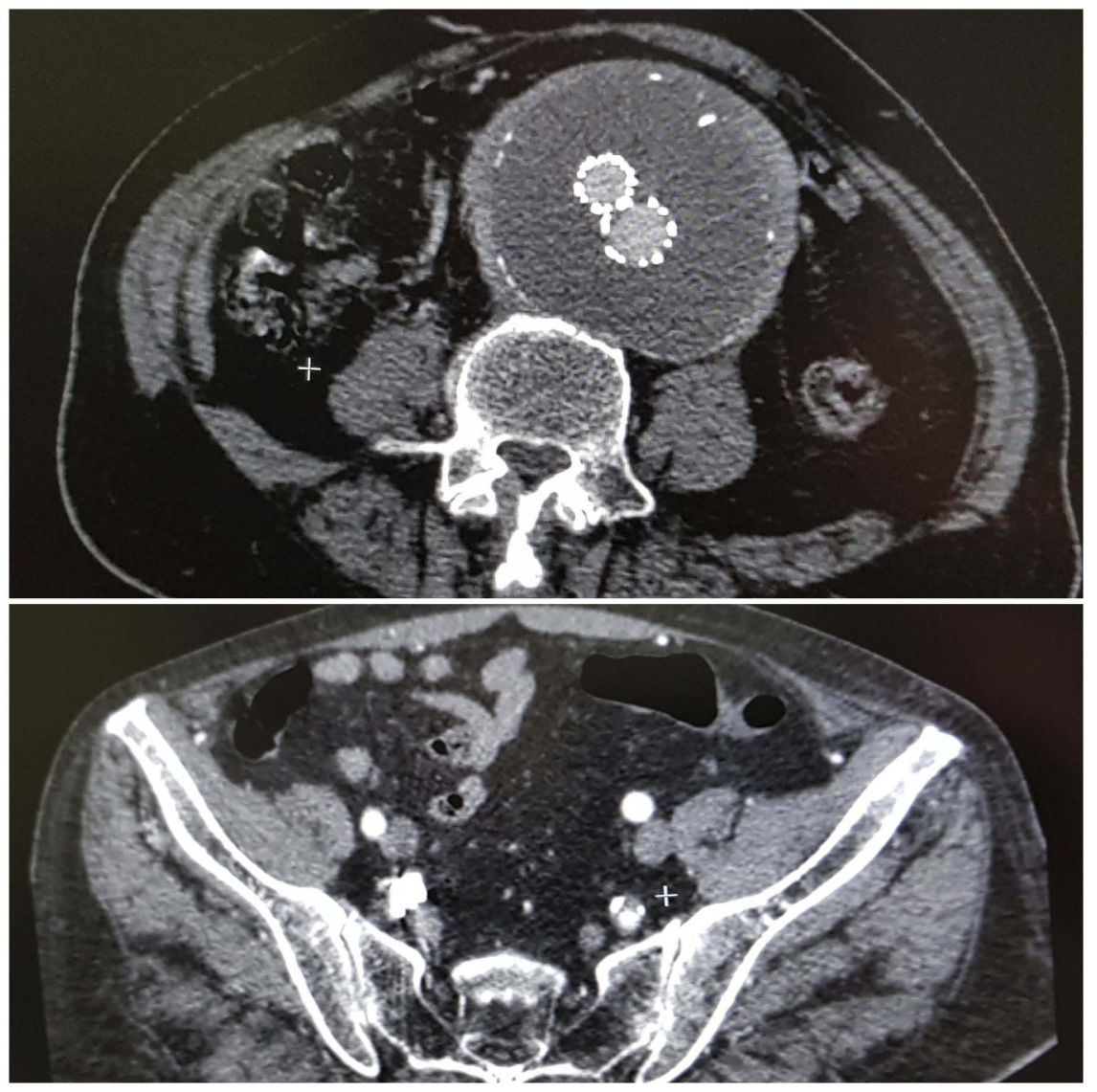

Figure 4 Follow-up CTA showing: (A) patent graft with exclusion of the aneurysm from circulation and without endoleak, Magnification XI. (B) Retrograde flow into RIIA, Magnification XI.

Abbreviations: CTA, Computed Tomography Angiography; RIIA, Right Internal Iliac Artery

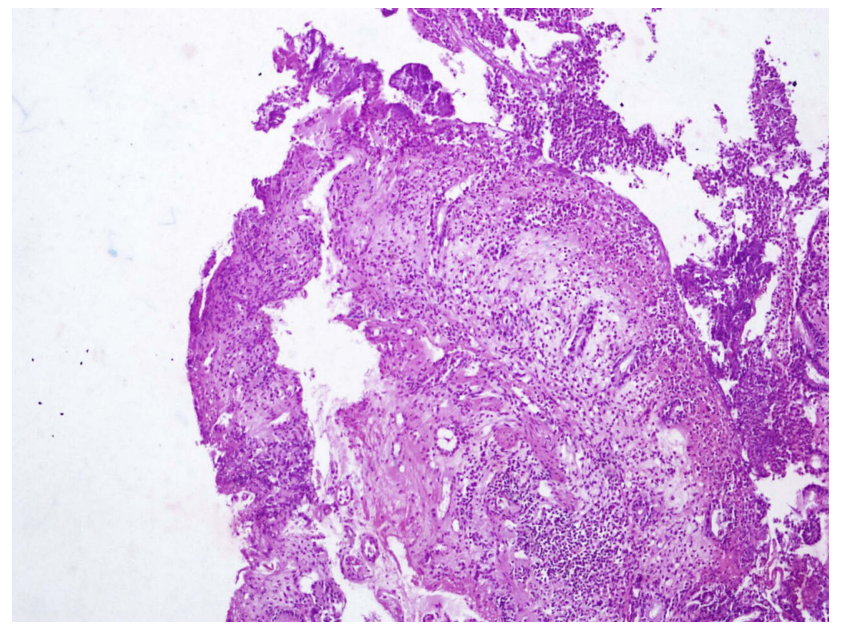

Figure 5 Ischemic colitis changes seen on a rectal biopsy. Magnification X4

The current management of SCI following EVAR is evolving. ${ }^{2,4}$ Various neuroprotective interventions have contributed to improved neurological outcomes following surgical or endovascular aortic interventions. ${ }^{2,5,9,11,12}$
Technical and pharmacological measures with favorable outcomes include CSF drainage, staged repairs, iliac branched devices, increasing mean arterial pressure, steroid administration, hyperbaric oxygen, naloxone, papaverine hydrochloride, and barbiturates.

The existing body of literature reported three cases of significantly delayed ( $>2$ weeks) SCI following EVAR: Reid et al reported a delayed, but temporary paraplegia 2 weeks following EVAR, ${ }^{13}$ Goldstein et al reported a delayed, but permanent paraplegia 3 weeks following EVAR, ${ }^{9}$ whereas Maddock et al reported a delayed, but reversible hemiparesis 8 weeks following EVAR. ${ }^{7}$ Interestingly, all three cases demonstrated acute hypoperfusion of the spinal cord secondary to hypotension.

In the present case, the etiology of delayed SCI is likely multifactorial. The patient had significant spinal stenosis, which may worsen spinal perfusion pressure in the presence of reperfusion edema within a confined compartment. Additionally, the patient was prescribed 
a potent alpha-blocker, which resulted in postural hypotension and reduced mean arterial pressure. The unique delayed presentation of SCI in this patient was precipitated by hemodynamic instability resulting from the use of alpha-blockers in the presence of vulnerable pelvic circulation.

Substantial evidence highlights the importance of maintaining adequate perioperative BP in aortic reconstructions. Safi et al reported that $50 \%$ of cases presenting with delayed SCI following thoracoabdominal aneurysm repair had a period of BP instability. ${ }^{14}$ Melissano et al advocated suspending antihypertensive medications following complex aortic reconstruction to avoid postoperative hypotension and hemodynamic instability. ${ }^{2}$ Moreover, Scott et al emphasized the importance of maintaining adequate arterial pressure regardless of hospitalization status. ${ }^{11}$

Our case report highlights the importance of maintaining adequate hemodynamic stability by augmenting arterial BP in cases of marginal pelvic vascular insufficiency. In our case, CSF drainage was avoided because the patient was treated with dual antiplatelet therapy and had significant spinal stenosis. Furthermore, recent evidence suggests internal iliac plugging is preferable to coil embolization if required in EVAR. ${ }^{15}$

To the best of our knowledge, this is the first reported case of significantly delayed isolated fecal incontinence following EVAR that has been successfully reversed by conservative management.

In conclusion, alpha-blockers are best avoided in cases of marginal vascular insufficiency of the spinal cord following aortic interventions. Vulnerability to $\mathrm{SCI}$ is not limited to the perioperative period but persists following patient discharge. Finally, hemodynamic SCI is potentially reversible if managed promptly and efficiently.

\section{Ethics statement and patient consent}

Written informed consent was obtained from the patient for publication. Institutional approval was not required.

\section{Abbreviations}

SCI, spinal cord ischemia; AAA, abdominal aortic aneurysm; EVAR, endovascular aneurysm repair; HBO, hyperbaric oxygen; DRE, digital rectal examination; BP, blood pressure.

\section{Acknowledgments}

This research did not receive any specific grant from funding agencies in the public, commercial, or not-forprofit sectors.

\section{Disclosure}

The authors report no conflicts of interest in this work.

\section{References}

1. Moulakakis KG, Alexiou VG, Karaolanis G, et al. Spinal cord ischemia following elective endovascular repair of infrarenal aortic aneurysms: a systematic review. Ann Vasc Surg. 2018;52:280-291. doi:10.1016/j.avsg.2018.03.042

2. Melissano G, Bertoglio L, Mascia D, et al. Spinal cord ischemia is multifactorial: what is the best protocol? J Cardiovasc Surg (Torino). 2016;57(2):191-201.

3. Berg P, Kaufmann D, van Marrewijk CJ, Buth J. Spinal cord ischaemia after stent-graft treatment for infra-renal abdominal aortic aneurysms. Analysis of the eurostar database. Eur J Vasc Endovasc Surg. 2001;22(4):342-347. doi:10.1053/ejvs.2001.1470

4. Fortes DL, Atkins BZ, Chiou AC. Delayed paraplegia following infrarenal abdominal aortic endograft placement: case report and literature review. Vascular. 2004;12(2):130-135. doi:10.1258/ rsmvasc.12.2.130

5. Riess KP, Gundersen SB, Ziegelbein KJ. Delayed neurologic deficit after infrarenal endovascular aortic aneurysm repair. Am Surg. 2007;73(4):385-387.

6. Bhama JK, Lin PH, Voloyiannis T, Bush RL, Lumsden AB. Delayed neurologic deficit after endovascular abdominal aortic aneurysm repair. J Vasc Surg. 2003;37(3):690-692. doi:10.1067/mva.2003.161

7. Maddock MJ, Modi S, Nicholl P, Wee B. Lumbar sacral plexopathya rare and late complication of endovascular aneurysm repair. $J$ Vasc Interv Radiol. 2013;24(3):448-449. doi:10.1016/j.jvir.2012.12.012

8. Lioupis C, Tyrrell M, Valenti D. A report of spinal cord ischemia following endovascular aneurysm repair of an aneurysm with a large thrombus burden and complex iliac anatomy. Vasc Endovascular Surg. 2010;44(1):56-60. doi:10.1177/1538574409345031

9. Goldstein LJ, Rezayat C, Shrikhande GV, Bush HL. Delayed permanent paraplegia after endovascular repair of abdominal aortic aneurysm. J Vasc Surg. 2010;51(3):725-728. doi:10.1016/j. jvs.2009.09.023

10. Awad H, Bratasz A, Nuovo G, et al. MiR-155 deletion reduces ischemia-induced paralysis in an aortic aneurysm repair mouse model: utility of immunohistochemistry and histopathology in understanding etiology of spinal cord paralysis. Ann Diagn Pathol. 2018;36(8):12-20. doi:10.1016/j.anndiagpath.2018.06.002

11. Scott D, Denton M. Spinal cord protection in aortic endovascular surgery. BJA. 2016;117(suppl_2):ii26-ii31. doi:10.1093/bja/aew217

12. Nishioka N, Kurimoto $\mathrm{Y}$, Maruyama R, et al. Bladder and rectal incontinence without paraplegia or paraparesis after endovascular aneurysm repair. Surg Case Rep. 2016;2(1):11. doi:10.1186/s40792016-0140-y

13. Reid JA, Mole DJ, Johnston LC, Lee B. Delayed paraplegia after endovascular repair of abdominal aortic aneurysm. J Vasc Surg. 2003;37(6):1322-1323.

14. Safi HJ, Miller III CC, Azizzadeh A, Iliopoulos DC. Observations on delayed neurologic deficit after thoracoabdominal aortic aneurysm repair. J Vasc Surg. 1997;26(4):616-622.

15. Bosanquet DC, Wilcox C, Whitehurst L, et al. Systematic review and meta-analysis of the effect of internal iliac artery exclusion for patients undergoing EVAR. Eur J Vasc Endovasc Surg. 2017;53 (4):534-548. doi:10.1016/j.ejvs.2017.01.009 


\section{Publish your work in this journal}

Vascular Health and Risk Management is an international, peerreviewed journal of therapeutics and risk management, focusing on concise rapid reporting of clinical studies on the processes involved in the maintenance of vascular health; the monitoring, prevention and treatment of vascular disease and its sequelae; and the involvement of metabolic disorders, particularly diabetes. This journal is indexed on PubMed Central and MedLine. The manuscript management system is completely online and includes a very quick and fair peerreview system, which is all easy to use. Visit http://www.dovepress. com/testimonials.php to read real quotes from published authors.

Submit your manuscript here: https://www.dovepress.com/vascular-health-and-risk-management-journal 